\title{
Aide à la replanification d'un projet avec réservation de créneaux de ressources
}

\author{
Caroline Thierry*,** - Jacques Lamothe ${ }^{* * *}$ \\ Vincent Galvagnon $* *, * * * *$
}

* Université Toulouse II Le Mirail, Dpt Mathématiques et informatique

5, allée Antonio Machado, F-31058 Toulouse Cedex

thierry@univ-tlse2.fr

** ONERA-DCSD, 2, av. Édouard Belin, BP 4025, F-31055 Toulouse Cedex

*** Ecole des Mines d'Albi Carmaux, Centre de Génie Industriel,

Campus Jalard, F-81013 Albi Cedex 09

jacques.lamothe@enstimac.fr

**** Access Commerce, Le stratège, Bât B2, rue Ampère BP 555

F-31674 Labège Cedex

\begin{abstract}
RÉSUMÉ. Dans cet article, on s'intéresse à la planification d'un projet nécessitant des ressources unitaires, non divisibles et jugées critiques qui, sur certains créneaux de temps, ont déjà été réservées par d'autres projets ou d'autres entreprises. A partir d'une expression initiale d'un réseau d'activités, le rôle d'un responsable de la planification est soit de fournir un ordonnancement réalisable de ces activités, soit de redéfinir le réseau pour pouvoir trouver ensuite un ordonnancement réalisable. Dans ce cadre, nous proposons un outil d'aide à la replanification, dont le prototype a été testé sur une application industrielle, destiné au responsable d'un projet en intégrant les contraintes de réservation de ressources liées au contexte multiprojet, multi-entreprise. Cet outil intègre une méthode qui, lorsque le problème est incohérent, exhibe les causes de cette incohérence et lorsque la cohérence a été restaurée propose une solution au problème d'ordonnancement.

ABSTRACT. In this paper we focus on the replanning process of a single project which uses unitary, undivisible resources that are considered critical. Because of other projects or other companies, those ressources are booked on several time-windows. From an inital problem definition, at the beginning of the project, the planning work essentially consists in updating the problem definition itself, during all of the project life, when unfeasibility arises due to disturbances. More over the multi-project is also to be considered through the booked resources constraints. We propose a decision support system, tested on an industrial case, for a project manager, which incorporates the constraints of the booked resources due to the multi-project and multi-company context. This system displays explanations for problem inconsistency likely to help the decision-maker to restore consistency and proposes a solution to the project-scheduling problem when the consistency has been restored.

MOTS-CLÉS : planification, gestion de projet, ordonnancement.

KEYWORDS: Decision Support System, Planning, Project Management, Task Scheduling.
\end{abstract}




\section{Introduction}

\subsection{Description du contexte}

Traditionnellement, la production de biens dans les entreprises fait l'objet d'une planification à long, moyen et court terme. La planification à moyen terme dans le cadre d'une production en série est axée sur l'optimisation du processus de fabrication en termes de ressources et de quantités produites. Dans le cadre de la production en série unitaire, du fait de la complexité de réalisation des produits concernés (innovants ou de haute technicité), les entreprises utilisent une structure de projet pour gérer leur production (Giard, 1991 ; Giard et al., 1996 ; PMI 1994).

Pour certaines de leurs activités, ces projets font généralement appel à des ressources unitaires, renouvelables, non divisibles et dites lourdes car jugées critiques (salle blanche, banc de test pour la production de satellites). Ce sont souvent des ressources de haute technologie, en quantité faible, ayant une faible disponibilité et qu'il s'agit de réserver longtemps à l'avance. Dans une entreprise, plusieurs projets coexistent, à des degrés d'avancement divers et doivent partager ces ressources. Par ailleurs, il faut noter que les ressources lourdes peuvent être réservées auprès de fournisseurs externes et ouvertes à des entreprises extérieures. Les projets de production unitaire peuvent donc être portés par des entreprises différentes, voire même par des entreprises concurrentes. Etant donnés la dimension de ces projets (en nombre de tâches, en nombre d'acteurs, en coût, en durée et en enjeu) et le contexte multi-entreprise, nous considérons une organisation où chaque projet est géré de manière indépendante même s'il partage des ressources avec d'autres projets du même type et est contraint par le comportement de ses fournisseurs (disponibilité des équipements) et de ses clients (jalons, dates d'exigibilité).

Chaque responsable de projet doit donc planifier son projet en considérant que les ressources lourdes ne sont disponibles que sur certains créneaux de temps : les créneaux déjà réservés par d'autres projets ou d'autres entreprises sont, en effet, difficilement renégociables.

\subsection{Description du problème}

Dans le contexte présenté ci-dessus, le problème qui nous concerne est celui de la replanification à moyen terme d'un projet, qui partage des ressources renouvelables de capacité unitaire, non divisibles avec différents projets. Ces ressources sont jugées suffisamment critiques par le responsable du projet pour qu'il souhaite se positionner par rapport à leur disponibilité sur le moyen terme. Il ne s'agit pas de modifier le séquencement de l'ensemble des projets sur ces ressources partagées car le décideur ne dispose que de l'accès aux données concernant son projet. L'objectif est plutôt de replanifier, reséquencer ou redéfinir un projet en tenant compte des 
contraintes imposées par les autres projets du fait du partage des ressources critiques.

On se pose alors le problème de la résolution d'un problème d'ordonnancement dont les contraintes de ressources portent sur l'impossibilité d'effectuer une tâche sur les créneaux de ressource occupés par d'autres projets (figure 1). On parlera de « problème d'ordonnancement avec réservation de créneaux de ressources ».

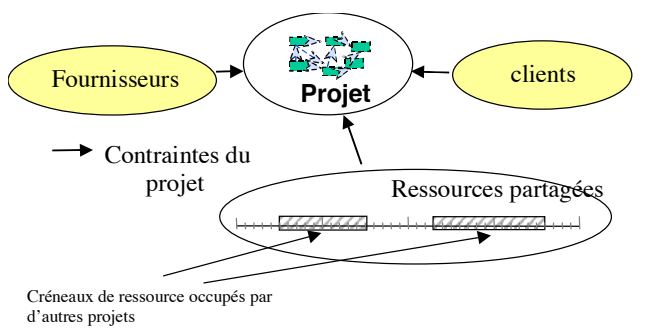

Figure 1. Contraintes d'un projet dans un contexte multiprojet, multi-entreprise

En adoptant cette vision locale consacrée à un projet, l'objectif est de fournir une aide locale au responsable de projet en ce qui concerne la planification des tâches dans ce contexte multiprojet. Cette aide peut se situer à deux niveaux : s'il existe une (ou plusieurs) solution(s) il s'agit de la (les) fournir à l'utilisateur. S'il n'existe pas de solution, il s'agit de lui fournir une aide à la résolution des conflits en lui laissant la responsabilité de sa décision. Il ne s'agit donc pas de déterminer comment modifier le séquencement de l'ensemble des projets sur les ressources mais de permettre au décideur d'exploiter les leviers d'actions qu'il maîtrise. Ces leviers sont essentiellement de deux types : ceux qui restent dans le cadre de l'autonomie de son projet et ceux qui nécessitent une négociation avec les autres acteurs impliqués (autres projets, fournisseurs ou donneurs d'ordres). Parmi les leviers relevant de la première catégorie on peut citer un changement de séquence entre deux tâches du projet ou une diminution de la durée d'une tâche (ajout d'une équipe). Parmi ceux qui sont conditionnés à une négociation préalable on peut citer l'anticipation d'une livraison par un fournisseur, le report d'une livraison ou encore une libération par un autre projet d'un créneau de ressource.

Généralement, l'entreprise ou le responsable de projet a acquis un savoir-faire qui permet de décomposer a priori le projet et de déterminer un ensemble de tâches à réaliser et un ordre partiel sur les tâches. Cependant, ces tâches, tout comme certaines contraintes entre ces tâches, ne sont pas uniquement fixées par un processus de production rigide. Il est en général possible de modifier des tâches (par exemple, de façon à réduire leur durée) et de modifier la façon dont elles s'enchaînent (par exemple, en inversant deux tests...). Ainsi on dispose d'un problème de base (un ensemble de tâches utilisant des ressources et un ordre partiel 
sur les tâches du projet) qui définit un problème d'ordonnancement, mais on sait que les données mêmes du problème ainsi que les contraintes pourront être modifiées si besoin est.

Dans le travail présenté ici, seules les contraintes de partage des ressources matérielles entre plusieurs projets ont été prises en compte. Les autres contraintes de ressources (ressources humaines ou ressources internes) entre des tâches d'un même projet ne sont pas considérées dans le processus de planification. Par ailleurs, on s'intéresse à des ressources très spécifiques qui ne sont utilisées que par un nombre très faible de tâches d'un projet, voire même par une seule tâche. Il n'existe donc pas de contraintes disjonctives entre deux tâches du même projet: lorsque plusieurs tâches utilisent une même ressource, la contrainte est gérée par le décideur qui résout la contrainte disjonctive en faisant un arbitrage qui se traduit par l'ajout de contraintes de précédence entre les tâches concernées. Il utilise alors le savoir-faire dont il dispose sur la famille de biens à laquelle le produit concerné appartient.

\subsection{Approche adoptée}

Le but de cette étude est de concevoir un outil d'aide à la replanification destiné au gestionnaire de projet qui est confronté au problème «d'ordonnancement moyen terme du projet avec créneaux de ressources ». Cet outil adopte le point de vue du décideur en charge d'un seul projet soumis à des contraintes liées au contexte multiprojet. La principale difficulté est l'aide à la replanification lorsque le problème d'ordonnancement avec réservation de ressources posé n'a pas de solution.

Notre objectif est de proposer au décideur des explications à cette non-existence de solution pour qu'il puisse modifier le problème et obtenir une solution (figure 2)

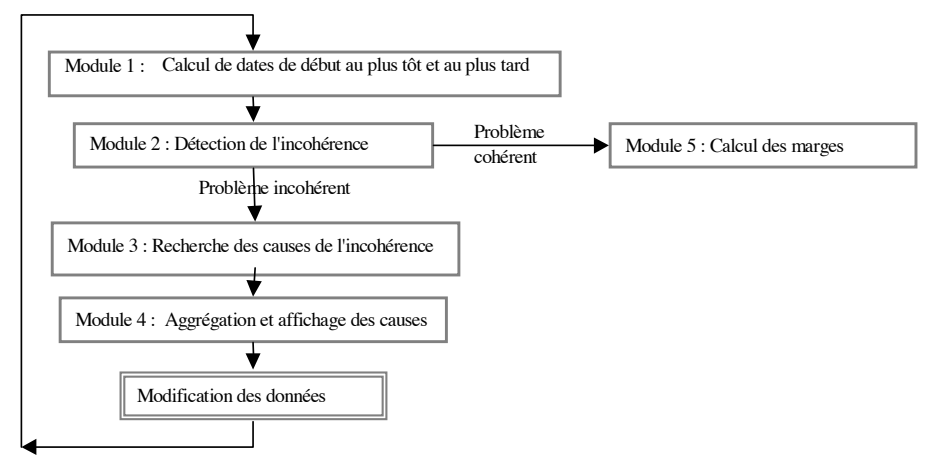

Figure 2. Structure de l'outil d'aide à la replanification

Dans la suite de cet article, après avoir présenté un état de l'art sur les problèmes d'assemblage à la commande et d'ordonnancement multiprojet, nous présenterons, 
les caractéristiques du problème d'ordonnancement avec réservation de ressources. Puis nous montrerons comment déterminer les dates de début au plus tôt, les dates de début au plus tard et les marges des tâches, en relâchant tour à tour les contraintes de dates d'exigibilité puis les contraintes de dates de disponibilité.

Dans un deuxième temps, nous montrerons comment détecter puis expliquer l'incohérence dans le cas où le problème posé n'a pas de solution. En effet, dans ce cas, on se propose alors de répondre à la question «pourquoi ca ne marche pas? » et de donner au décideur des indications sur «quoi modifier pour obtenir une solution ? ».

\section{Etat de l'art}

\subsection{Assemblage à la commande}

Dans le cas de la production à la commande dans le contexte industriel «toutes les matières premières et les composants doivent être fournis au moment de la réception de la commande client » (Kolisch, 2001).

Pour la planification de la production dans le domaine de la production à la commande, nous différentions selon la répétitivité du flux les contributions concernant l'ordonnancement et la planification multiniveau : «multi-level scheduling and lot sizing » (Maes et al., 1991 ; Drexl et al., 1997 ; Dauzère-Perès et al., 1994) de celles concernant la gestion multiprojet. Comme chaque processus d'assemblage peut être considéré comme un projet, les modèles et méthodes d'ordonnancement de projet ont été largement utilisés pour la production à la commande. De plus, comme généralement plusieurs processus d'assemblage ont lieu simultanément, on parle d'ordonnancement multiprojet.

\subsection{Ordonnancement de projet}

Dans le cadre de la gestion de projet, les techniques d'ordonnancement (Kelley, 1961 ; Kolisch et al., 2001) sont utilisées pour déterminer les dates de début des tâches en fonction de contraintes technologiques et des contraintes de ressources. De nombreuses études (Weglarz 1999; Brucker et al., 1999 ; Kolisch et al., 2001 ; Hartmann et al., 1998) se sont intéressées à la gestion de ressources renouvelables cumulatives (RCPSP : Resource-Constrained Project Scheduling Problem). Dans ce type de problème (RCPSP), chaque tâche du projet nécessite une certaine quantité de ces ressources cumulatives. De nombreuses méthodes d'optimisation ou des méthodes heuristiques ont été développées pour résoudre ce type de problème. (Kolisch et al., 1999) présentent une classification et une analyse des performances de ces études. Récemment, des techniques de recherche locales ou metaheuristiques (recuit simulé, recherche tabu et algorithmes génétiques) ont été employées pour ces problèmes avec des résultats prometteurs. Par ailleurs, des méthodes de type 
propagation de contraintes ont aussi été utilisées pour la résolution du RCPSP (contraintes disjonctives ou cumulatives) ou pour le problème de l'ordonnancement de type job-shop (Nuijen, 1994 ; Dorndorf et al., 1999).

De nombreuses études ont aussi porté sur la révision d'un réseau d'activité pour respecter des dates jalons (Giard, 1991). L'approche multimode considère que le coût et la durée de toute activité dépend du choix d'un mode d'affectation des ressources par activité. Des méthodes optimales ou heuristiques (Salewskia et al., 1997, Boctor 1996, Elmaghraby 1977) sont alors possibles pour sélectionner ces modes. D'autres approches proposent de modéliser plusieurs alternatives de décomposition d'un réseau d'activité (Crowston, 1971 ; Golenko et Blokh, 1997). Toutes ces approches considèrent par contre qu'il n'existe pas de limitation sur les dates auxquelles les activités peuvent être réalisées.

Si l'on considère des créneaux de temps, (Chen et al., 1997) ont étudié l'ordonnancement au plus tôt et au plus tard dans un réseau d'activité sans ressources lorsque les activités ne sont réalisables que sur une fenêtre de temps donnée ou à certaines dates figées. Ils généralisent alors le calcul de certaines marges temporelles dans ces cas.

\subsection{Ordonnancement multiprojet}

La gestion multiprojet s'intéresse pour sa part à un ensemble de projets afin d'optimiser l'utilisation des ressources qui sont généralement des ressources cumulatives. Lorsque des ressources sont partagées entre ces projets on parle de gestion multiprojet avec contraintes de ressources: resource-constrained multiproject scheduling problem (RCMPSP). Dans ce contexte, des méthodes d'ordonnancement basées sur des règles de priorité, ont été adaptées.

(Kurtulus et al., 1985) ont étudié l'efficacité de règles spécifiques avec une approche centralisée (i.e. MAXTWK : Maximum Total Work Content, qui est basé sur l'utilisation des ressources). D'autre part, (Yang et al., 1997) proposent différents types de règles utilisables dans une approche distribuée (MINPDD, une règle d'allocation de ressources donnant la priorité au projet dont la date due est minimale). Les approches distribuées du problème d'ordonnancement multiprojet utilisent un modèle hiérarchique qui décompose le problème en plusieurs sousmodèles. (Speranza et al., 1993) utilisent une approche d'optimisation multicritère de la planification et l'ordonnancement multiprojet avec une hiérarchie de trois modèles linéaires en nombres entiers. Le premier modèle donne les modes d'exécution macroscopiques, le second modèle alloue les ressources aux projets ; enfin le modèle détaillé ordonnance les tâches du projet.

Le problème que nous développons par la suite fait apparaître des contraintes de ressources dans un projet qui sont dues à son environnement dans un contexte multiprojet. Les autres projets imposant des créneaux de réservation sur les ressources partagées, nous considérons un contexte bien particulier de gestion d'un 
projet soumis à des contraintes dues à l'environnement multiprojet et multientreprise. Notre problématique se rapproche plutôt de celle développée par (Chen et $a l ., 1997)$ à la différence près qu'un ensemble de créneaux temporels est proposé pour chaque activité. Nous montrerons de même que la notion de marge doit être interprétée dans le détail, ce qui augmente la complexité des approches pour réviser le réseau d'activités.

Après une description du problème d'ordonnancement de projet étudié, nous détaillons l'outil d'aide à la replanification permettant de détecter l'incohérence du problème (grâce au calcul des dates de début au plus tôt et au plus tard) et de donner des causes de l'incohérence pour une aide à la restauration d'une solution et donc à la replanification.

\section{Ordonnancement de projet avec réservation de créneaux de ressources}

\subsection{Caractéristiques du problème}

$\mathrm{Au}$ niveau de chacun des projets, le problème d'ordonnancement de projet avec réservation de créneaux de ressources (dit BRPSP pour Booked Resource ProjectScheduling Problem) fait partie de la classe de problèmes dits Resource-Constrained Project-Scheduling Problem (RCPSP). La caractéristique principale de ce problème est le fait que les ressources considérées sont des ressources renouvelables dont certains créneaux de temps ont été réservés par d'autres projets. Le problème d'ordonnancement de projet avec réservation de créneaux de ressources consiste donc à placer les tâches dans les créneaux disponibles.

Si on utilise la classification de ces problèmes proposée dans (Herroelen et al., 1999), les tâches du problème d'ordonnancement étudié sont soumises à :

- des contraintes de précédence (fin-début avec décalage de valeur 0),

- des contraintes de disponibilité liées à l'approvisionnement de composants ou de sous-ensembles,

- des contraintes de dates d'exigibilité liées à des jalons fixés par les clients,

- des contraintes de ressources renouvelables de capacité 1 . Du fait de l'approche adoptée, ces contraintes de ressources se traduisent par des interdictions quant à l'utilisation de certains créneaux de temps réservés par les autres projets pour l'exécution des tâches concernées.

Par ailleurs, la préemption n'est pas autorisée et les contraintes de changement de série sont incluses dans les durées des tâches.

\subsection{Modèle}

Nous proposons une formulation du problème d'ordonnancement considéré sous la forme d'un problème de satisfaction de contraintes. Soit $I$ le nombre de tâches, 
Le problème $P=\left(V, D, C, P_{i}, P_{e}\right)$ est constitué par :

- l'ensemble $V$ des variables : les date de début $t_{i}$ des tâches $T_{i}(i \in\{1,2, \ldots, I\})$. Soit $t_{i}$ la date de début au plus tôt de la tâche $T_{i}$ et $\bar{t}_{i}$ sa date de début au plus tard. $\underline{t_{i}}$ et $\bar{t}_{i}$ sont respectivement la borne minimale et la borne maximale de $t_{i}$;

- l'ensemble $D$ des domaines des variables de $V$ qui donne pour chaque variable un domaine $D_{i}$ de valeurs autorisées. $D_{i}$ est considéré comme une union de créneaux indépendants : $D_{i}=\bigcup_{x \in\left[1, n_{i}\right]}\left[S D_{x}^{i}, E D_{x}^{i}\right]$. Initialement: $\mathrm{n}_{\mathrm{i}}=1, \mathrm{SD}^{\mathrm{i}}=-\infty$ et $\mathrm{ED}_{1}^{\mathrm{i}}=+\infty$. Nous verrons ensuite comment ses domaines évoluent du fait des contraintes de ressources ;

- l'ensemble $P_{i}$ des paramètres internes: la durée $d_{i}$ et les ressources $R(i)$ nécessaires pour toute tâche $T i$;

- l'ensemble $P_{e}$ des paramètres externes (relative à l'environnement extérieur du projet). Ils sont en général négociables avec les fournisseurs, les autres projets voire même les clients. L'ensemble $P_{e}$ comprend :

- les dates d'arrivées des approvisionnements, qui sont nécessaires pour l'exécution de la tâche $T_{i}: A_{i}$

- les dates d'exigibilité (jalons imposés par le client) de la tâche $T_{i}: J_{i}$

- les réservations $O_{m}$ de chaque ressource $\left.R_{m}: \quad O_{m}=\bigcup_{x \in\left[1, n_{m}\right]}\right] S_{x}^{m}, E_{x}^{m}[$

Ces réservations sont données pour chaque $R_{m}$, sous la forme d'un ensemble d'intervalles $] S_{x}^{m}, E_{x}^{m}$ [ (chaque intervalle correspondant à l'utilisation d'une ressource par un autre projet et qui commence à $S_{x}^{m}$ et se termine à $E_{x}^{m}$ ).

- l'ensemble $\mathrm{C}$ des contraintes qui lient les variables aux paramètres :

- les contraintes temporelles :

$$
\mathrm{t}_{0}=0
$$

$\forall \mathrm{i}, \mathrm{t}_{\mathrm{i}}-\mathrm{t}_{0} \geq \mathrm{A}_{\mathrm{i}}$

$$
(\mathrm{i}=1, \ldots, \mathrm{I})
$$$$
(i=1, \ldots, I ; j=1, \ldots, I)
$$

$$
\forall \mathrm{i}, \mathrm{t}_{\mathrm{i}}+\mathrm{d}_{\mathrm{i}} \leq \mathrm{J}_{\mathrm{i}}
$$$$
(\mathrm{i}=1, \ldots, \mathrm{I})
$$

La contrainte [1] assure qu'aucune tâche ne commence avant le début du projet $t_{0}$. Les contraintes de disponibilité [2] imposent à la tâche de ne commencer qu'après 
une date donnée (arrivée des approvisionnements) $A_{i}$. Les contraintes de précédence [3] qui imposent un ordre entre les tâches et qui sont le résultat du choix d'une séquence d'assemblage par le décideur. Certaines de ces contraintes sont des contraintes technologiques, d'autres sont le résultat d'une décision de séquencement de tâches a priori, reflétant l'expertise du décideur, d'autres sont issus de la résolution par le décideur de contraintes de ressources disjonctives entre les tâches du projet. Les contraintes de dates d'exigibilité [4] imposent à la tâche de finir avant une date $J_{i}$ fixée par le client.

- les contraintes de ressources

Les ressources considérées sont partagées avec d'autres projets. Une tâche donnée ne peut être exécutée que si elle peut accéder à un créneau de ressource libre correspondant à ses besoins (figure 1). Donc la contrainte sur le domaine de la date de début $t_{i}$ de la tâche $T_{i}$ qui utilise les ressources $R_{m} \in R(i)$ est la suivante :

$$
\left[t_{i}, t_{i}+d_{i}\right] \subset \bigcap_{m \in R(i)} \overline{O_{m}}
$$

Avec $\overline{O_{m}}$ le complémentaire de $O_{m}$. Donc :

$$
\left.\overline{O_{m}}=-\infty, S_{1}^{m}\right] \bigcup_{x \in\left[2, n_{m}\right]}\left[E_{x-1}^{m}, S_{x}^{m}\right] \bigcup\left[E_{n_{m}}^{m},+\infty[\right.
$$

De plus, les tâches sont considérées comme non préemptibles. Elles doivent être terminées dans le créneau où elles sont commencées.

Donc la contrainte d'occupation de créneaux de ressources pour la tâche $T_{i}$ qui utilise $R(i)$ est :

$$
\begin{aligned}
& t_{i} \in D_{i} \text { avec } \\
& D_{i}=\bigcap_{m \in R(i)}\left\{1-\infty, S_{1}^{m}-d_{i}\right] \bigcup_{x \in\left[2, n_{m}\right]}\left[E_{x-1}^{m}, S_{x}^{m}-d_{i}\right] \bigcup\left[E_{n_{m}}^{m},+\infty[\}\right.
\end{aligned}
$$

Etant données les ressources $R(i), D_{i}$ est une l'intersection d'une série d'unions d'intervalles indépendants (contrainte [7]). Cette intersection est calculée polynomialement en $O\left(\sum_{m \in R(i)} n_{m}\right)$ et est, elle-même, une union d'intervalles indépendants (voir figure 3) : $D_{i}=\bigcup_{x \in\left[1, n_{i}\right]}\left[S D_{x}^{i}, E D_{x}^{i}\right]$ où $S D_{1}^{i}=-\infty$ et $E D_{n i}^{i}=+\infty$. 


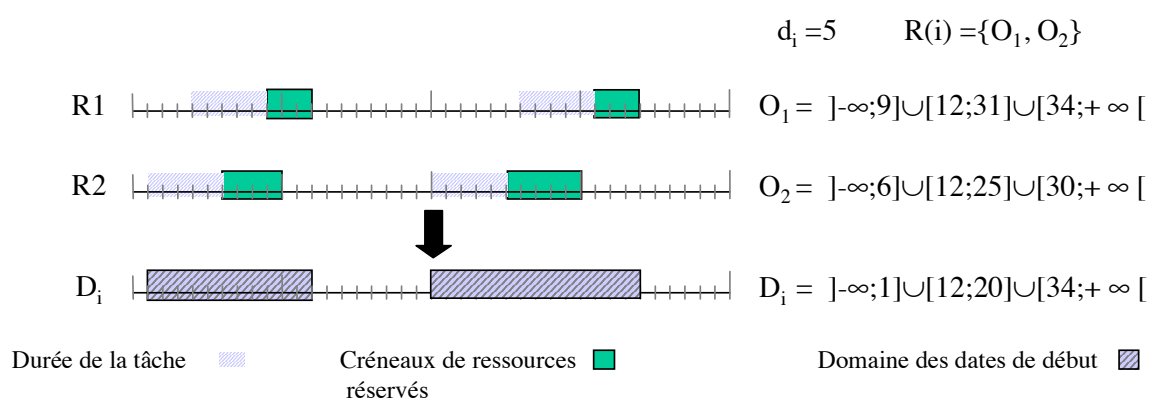

Figure 3. Exemple de créneaux de ressources réservés et domaine des dates de début des tâches résultant

A partir de cette formulation du modèle, on peut conclure que :

- les contraintes [1], [2], [3], [4] sont les contraintes classiques des problèmes d'ordonnancement de projet sans ressources : modèles PERT ou CPM classiques ;

- les contraintes liées au partage des ressources (relation [7]) sont vues par le projet comme des contraintes de créneau de temps pour le début des tâches. Par contre, (Chen et al., 1997) considèrent des contraintes sur les dates de début possibles proches mais différentes:

- contraintes de fenêtre temporelle,

- contraintes d'un ensemble fini de dates de début par tâche.

La contrainte de ressource (relation [7]) apparaît comme une généralisation des contraintes de (Chen et al., 1997). Nous chercherons à employer le même mode de raisonnement pour déterminer les dates de début au plus tôt et au plus tard.

\subsection{Représentation du problème par un réseau d'activité}

Par analogie avec un réseau PERT, ce type de problème peut être représenté par un réseau d'activité. Les nœuds du réseau représentant les dates de début des tâches et les arcs les contraintes de précédence entre les tâches ou les contraintes de dates de disponibilité. Les valeurs des arcs notées $a_{i j}$ sont donc positives. On note $\operatorname{Prec}(j)$ et $\operatorname{Succ}(j)$ l'ensemble des nœud respectivement prédécesseurs et successeurs d'un nœud $(j)$. Les contraintes unaires (qui ne portent que sur une variable du problème ici une date de début) représentent les contraintes de dates d'exigibilité, et les contraintes de ressources contraignant les tâches par des créneaux de temps libres (la figure 4 donne un exemple de graphe). Un chemin est donc ici plus complexe que dans un graphe potentiel-tâche classique dans la mesure où les décalages dus aux contraintes unaires doivent être pris en compte. Nous appelons le chemin de $(i)$ à $(j)$, noté $P_{\alpha}(i, j)$, une chaîne d'arc $\alpha$ allant du nœud $(i)$ au nœud $(j)$ au sens classique plus les contraintes unaires qui portent sur les nœuds le long de cette chaîne. 


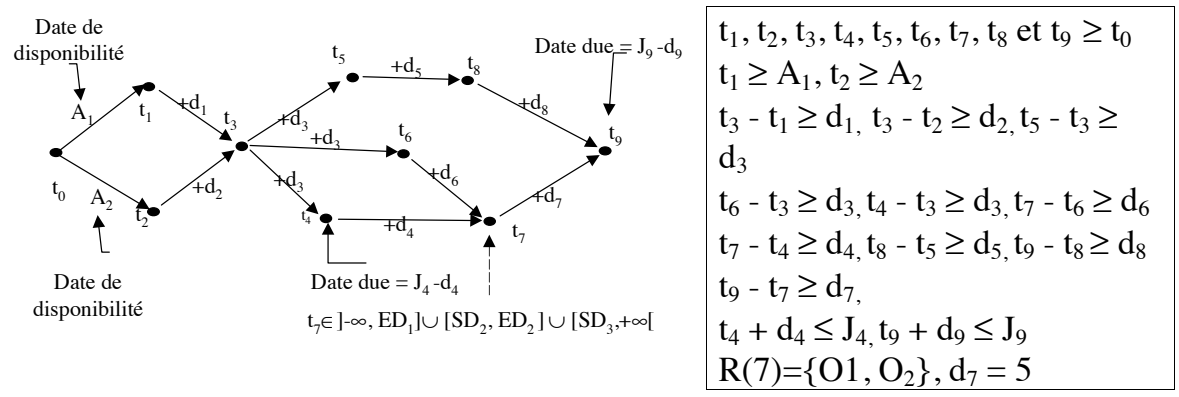

Figure 4. Exemple de réseau d'activité avec créneau de temps

De même que dans (Chen et al, 1997), on peut remarquer que si la date de début est donnée pour un prédécesseur $(i)$ d'un nœud $(j)$ :

- on peut calculer une date d'accès au nœud $(j): t_{i}+a_{i, j}$

- si cette date d'accès n'appartient pas à un créneau de ressource admissible, la contrainte de créneaux de ressource [7] décale la date de début de $(j)$ jusqu'au premier créneau disponible donc jusqu'à la date $S D_{x}^{j}$ qui est supérieure à la date d'accès.

On peut alors définir le décalage minimal entre $(i)$ et son successeur $(j)$ sachant que la tâche $T_{i}$ commence à $t_{i}$ et noté $\operatorname{DecTot}\left(i, j, t_{i}\right)$ :

$\operatorname{DecTot}\left(i, j, t_{i}\right)=\operatorname{MAX}\left[t_{i}+a_{i, j} \quad, \operatorname{Min}_{x \in\left[1, n_{j}\right]}\left(S D_{x}^{j} / E D_{x}^{j} \geq t_{i}+a_{i, j}\right)\right]-t_{i}$

La longueur du chemin entre deux sommets adjacents du réseau d'activité dépend donc de la date à laquelle il est considéré mais ne dépend que de ces deux sommets. On a donc la relation suivante entre $(j)$ et ses prédécesseurs :

$$
t_{j} \geq \operatorname{Max}_{i \in \operatorname{Pr} e d(j)}\left(t_{i}+\operatorname{DecTot}\left(i, j, t_{i}\right)\right)
$$

On notera que cette relation [9] comprend les contraintes ([2], [3], [7]) mais pas les contraintes [1] et [4].

Réciproquement, si la date de début est fixée pour un successeur $(j)$ d'un nœud (i), on peut calculer le décalage minimal entre $(i)$ et $(j)$ sachant que $(j)$ débute à la date $t_{j}$ noté $\operatorname{DecTard}\left(i, j, t_{j}\right)$ :

$$
\operatorname{DecTard}\left(i, j, t_{j}\right)=t_{j}-\operatorname{MIN}\left[\left(J_{i}-d_{i}\right),\left(t_{j}-a_{i, j}\right), \underset{x \in\left[1, n_{i}\right]}{\operatorname{Max}}\left(E D_{x}^{i} / S D_{x}^{i} \leq t_{j}-a_{i, j}\right)\right]
$$


On vérifie ici aussi que la longueur du chemin entre deux sommets adjacents dépend de la date considérée. Et notamment, un chemin considéré au plus tôt (selon DecTot) n'a pas la même longueur qu'un chemin considéré au plus tard (selon DecTard). On peut de même déduire la relation liant la date de début de $(i)$ à celle de ses successeurs :

$$
t_{i} \leq \operatorname{Min}_{j \in \operatorname{Succ}(i)}\left(t_{j}-\operatorname{DecTard}\left(i, j, t_{j}\right)\right)
$$

Cette relation [11] intègre toutes les contraintes sauf la contrainte [1].

Ainsi on notera ainsi qu'un problème BRPSP :

- peut être représenté par un graphe proche d'un graphe PERT ;

- peut être modélisé par des équations de récurrence liant la date de début d'un sommet à celle de ses prédécesseurs [9] ou de ses successeurs [11] si certaines contraintes sont relaxées. Ceci permet de vérifier les hypothèses de programmation dynamique classiques qui permettent le calcul des dates de début au plus tôt et au plus tard dans un réseau d'activité de type PERT;

- peut intégrer une notion de chemin qui doit être étendue pour intégrer les contraintes de ressource. La longueur d'un chemin dépendra par contre de la date à laquelle elle est considérée. Nous serons ainsi amenés a utiliser par la suite des types de longueur d'un chemin $P_{\alpha}(i, j)$ :

- $\operatorname{Lt} P_{\alpha}\left(i, j, t_{i}\right)$, longueur du chemin calculée lorsqu'on enchaîne les sommets au plus tôt sachant $t_{i}$ donné. Elle est définie récursivement par :

$$
\begin{aligned}
& L t P_{\alpha}\left(i, i, t_{i}\right)=0 \\
& L t P_{\alpha}\left(i, j, t_{i}\right)=\operatorname{Lt} P_{\alpha}\left(i, k, t_{i}\right)+\operatorname{Dec} \operatorname{Tot}\left(k, j, t i+L t P_{\alpha}\left(i, k, t_{i}\right)\right) \text { avec } k=\alpha \cap \operatorname{Pred}(j) .
\end{aligned}
$$

- $\operatorname{LTP}_{\alpha}\left(i, j, t_{j}\right)$, longueur du chemin calculée lorsque on enchaîne les sommets au plus tard sachant $\mathrm{t}_{\mathrm{j}}$ donné. Elle est définie par :

$$
\begin{aligned}
& \operatorname{LTP}_{\alpha}\left(j, j, t_{j}\right)=0 \\
& \operatorname{LTP}_{\alpha}\left(i, j, t_{i}\right)=\operatorname{LTP}_{\alpha}\left(k, j, t_{j}\right)+\operatorname{DecTard}\left(k, j, t_{j}-\operatorname{LTP}_{\alpha}\left(k, j, t_{j}\right)\right) \operatorname{avec} k=\alpha \cap \operatorname{Succ}(i)
\end{aligned}
$$

\subsection{Calcul des dates au plus tôt et des dates au plus tard}

L'objectif de cette étude est de proposer un outil d'aide à la replanification destiné aux responsables de projets qui adopte le point de vue d'un décideur (responsable d'un seul projet) en intégrant les contraintes liées au contexte multiprojet (partage de ressources, dates d'approvisionnement et de jalons).

Le calcul de la date de début au plus tôt de la tâche sera effectué en relaxant les contraintes de dates d'exigibilité (4). Sous cette hypothèse le problème BRPSP se résume à :

$$
t_{0}=0
$$




$$
t_{j} \geq \underset{i \in \operatorname{Pred}(j)}{\operatorname{aax}}\left(t_{i}+\operatorname{DecTot}\left(i, j, t_{i}\right)\right)
$$

La première étape du calcul de la date de début au plus tôt des tâches consiste à déterminer pour chaque tâche son domaine en fonction des ressources utilisées par la tâche et des dates de disponibilités qui la concernent. Ce domaine $\mathrm{D}_{\mathrm{i}}$ est défini par la relation [7]. Il faut noter que le dernier intervalle $\left[S D_{n i}^{i}, E D_{n i}^{i}\right.$ ] est tel que $E D_{n i}^{i}=+$ $\infty$. Ainsi, Si les contraintes de dates d'exigibilité [4] sont relâchées, les dates de début peuvent être décalées autant que nécessaire. Il existe ainsi toujours une date de début au plus tôt $t_{i}$ pour toute tâche $T_{i}$ (voir exemple figure 5).

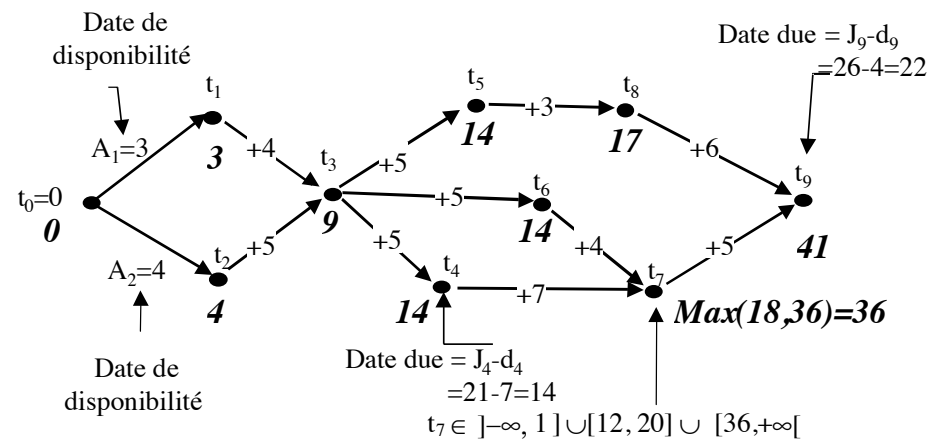

Figure 5. Exemple de calcul de dates de début au plus tôt

La relation [9] est une relation dynamique, entre un sommet et ses prédécesseurs, qui intègre toutes les contraintes non relaxées du problème. Les dates de début au plus tôt peuvent être calculées polynomialement en utilisant une procédure de propagation avant (Elmaghraby, 1977) et cette relation.

La valeur de $t_{i}$ est donc donnée par la formule suivante qui peut être déduite de la relation [9]:

$$
\underline{t_{0}}=0 \quad \text { et } \quad \underline{t_{j}}=\underset{i \in \operatorname{Pred}(j)}{\operatorname{Max}}\left(\underline{t_{i}}+\operatorname{DecTot}\left(i, j, \underline{t_{i}}\right)\right)
$$

On peut alors déterminer le chemin $P t_{c}(0, j)$ qui mène à la tâche $T_{j}$ en identifiant récursivement les prédécesseur dans la relation [12]. On trouve alors la relation entre la longueur au plus tôt du chemin critique et la date de début au plus tôt : $t_{j}=\operatorname{Lt} P_{P t c(0, j)}(0, j, 0)$.

L'ordonnancement au plus tôt étant obtenu en relaxant les contraintes de date due, il est une solution du problème d'ordonnancement avec réservations de ressources si et seulement si : $\forall i \in[0, I], \quad t_{i} \leq J_{i}-d_{i}$. 
Réciproquement, le premier intervalle $\left[S D_{1}^{i}, E D_{1}^{i}\right]$ de $D_{i}$ commence à $S D_{1}^{i}=-\infty$, il existe toujours un ordonnancement au plus tard qui respecte toutes les contraintes sauf celles de début du projet [1]. De plus, la relation [11] intègre toutes les contraintes du problème hormis la contrainte [1] et relie la date de début d'une tâche à celle de ses successeurs. Ainsi, les dates de début au plus tard des tâches peuvent aussi être calculées polynomialement par une procédure de propagation arrière (Elmaghraby, 1977) (voir exemple figure 6).

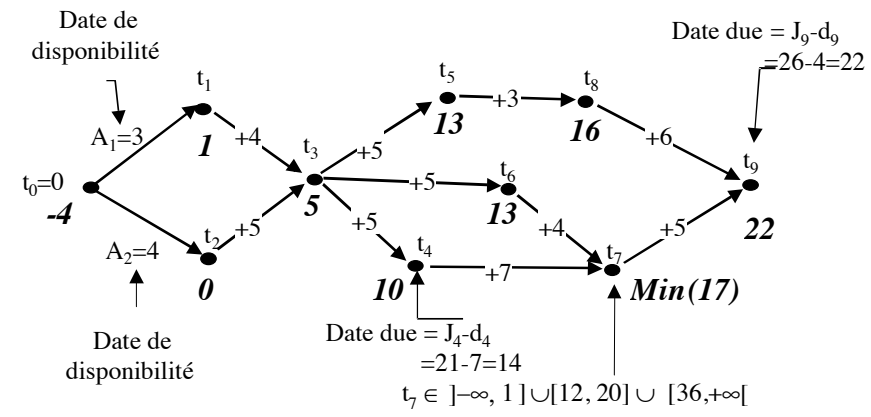

Figure 6. Exemple de calcul de dates de début au plus tard

La valeur de $\bar{t}_{i}$, est donnée par la formule suivante qui peut être déduite de la relation [11] :

$$
\overline{t_{i}}=\operatorname{Min}_{j \in \operatorname{Succ}(i)}\left(\overline{t_{j}}-\operatorname{Dec} \operatorname{Tard}\left(i, j, \overline{t_{j}}\right)\right)
$$

On pourra aussi identifier le chemin, noté $P T_{c}(i, j)$, entre une tache $T_{i}$ et un jalon sur une tache $T_{j}$ qui justifie sa date de début au plus tard. On retrouve alors la relation entre la longueur du chemin, le jalon et la date de début au plus tard :

$$
\overline{t_{i}}=J_{j}-d_{j}-\operatorname{LTP}_{P T C(i, j)}\left(i, j, J_{j}-d_{j}\right)
$$

Enfin, cet ordonnancement au plus tard est une solution du problème d'ordonnancement avec réservations de ressources, si et seulement si, il respecte la contrainte [1] soit : $\overline{t_{0}} \geq 0$

\subsection{Calcul des marges}

Lorsque l'ordonnancement au plus tôt et l'ordonnancement au plus tard ont été calculés, la date de début d'une tâche $T_{i}$ est contrainte par : $t_{i} \in\left[t_{i}, \bar{t}_{i}\right] \cap D_{i}$. Nous 
définissons ainsi de nouvelles notions de marges pour intégrer les effets dus aux ressources :

- marge totale : différence entre date au plus tard et date au plus tôt :

$$
M_{i}=t_{i}-\underline{t_{i}}
$$

Notons toutefois que deux tâches successives $\left(T_{j} \in \operatorname{Succ}\left(T_{i}\right)\right)$ appartenant à un même chemin critique n'auront pas forcément la même marge. En effet, comme $T_{i}$ et $T_{j}$ appartiennent au même chemin critique, on a :

$$
\overline{t_{i}}=\overline{t_{j}}-\operatorname{DecTard}\left(i, j, \bar{t}_{j}\right) \text { et } \underline{t_{j}}=\underline{t_{i}}+\operatorname{DecTot}\left(i, j, \underline{t_{i}}\right)
$$

$\operatorname{Donc} M_{j^{-}} M_{i}=\overline{t_{i}}-\underline{t_{i}}-\bar{t}_{j}+\underline{t_{j}}=\operatorname{DecTot}\left(i, j, \underline{t_{i}}\right)-\operatorname{DecTard}\left(i, j, \bar{t}_{j}\right) \neq 0$

Des tâches appartenant à un chemin critique peuvent ainsi avoir une marge totale positive. Mais aussi, un retard (ou une avance) qui consomme (rattrape) un peu de marge sur une tâche, peut en consommer (rattraper) beaucoup sur une autre.

- marge totale de liberté : marge temporelle réelle de début de la tâche. Elle se calcule selon la formule :

$$
M L_{i}=\left(\overline{t_{i}}-\underline{t_{i}}\right)-\sum_{x / E D_{x}^{i}>t_{\underline{i}} \text { and } S D_{x}^{i}<\overline{t_{i}}}\left(\operatorname{Min}\left(E D_{x}^{i}, \underline{t_{i}}\right)-\operatorname{Max}\left(S D_{x}^{i}, \overline{t_{i}}\right)\right)
$$

La somme $\sum_{x / E D_{x}^{i} x_{i} \text { and } S D_{x}^{i}<\overline{t_{i}}}\left(\operatorname{Min}\left(E D_{x}^{i}, \bar{t}_{i}\right)-\operatorname{Max}\left(S D_{x}^{i}, \overline{t_{i}}\right)\right)$ représente la somme des périodes de temps interdites pour le début de la tâche $T_{i}$ entre $\overline{t_{i}}$ and $\underline{t_{i}}$. Cela correspond au temps «perdu» entre $\bar{t}_{i}$ et $t_{i}$ du fait de la non-disponibilité des ressources.

Nous pouvons aussi étendre les notions de pré-attente et post-attente proposées dans (Chen et al., 1997) :

- pré-attente : temps écoulé entre la date de fin au plus tôt du dernier prédécesseur de la tâche et la date de début au plus tôt de la tâche :

$$
\left.\mathrm{pa}_{\mathrm{i}}=\underline{t_{i}}-\underset{j \in \operatorname{Pred}(i) \underline{\max }}{\left(t_{j}\right.}+d_{j}\right)
$$

La pré-attente est le temps perdu du fait de l'attente de la libération d'une ressource.

- post-attente : temps écoulé entre la date de fin au plus tard de la tâche et la plus petite des dates de début au plus tôt des successeurs de la tâche :

$$
P a_{i}=\underset{k \in \operatorname{Succ}(i)}{\operatorname{Min}}\left(\overline{\left.t_{k}\right)}-\overline{t_{i}}\right.
$$


La figure 7 représente ces notions de marges par rapport à la notion classique de marge totale. On voit ainsi que les notions de pré-attente, post-attente représentent des pertes de marge dues aux contraintes de ressource.

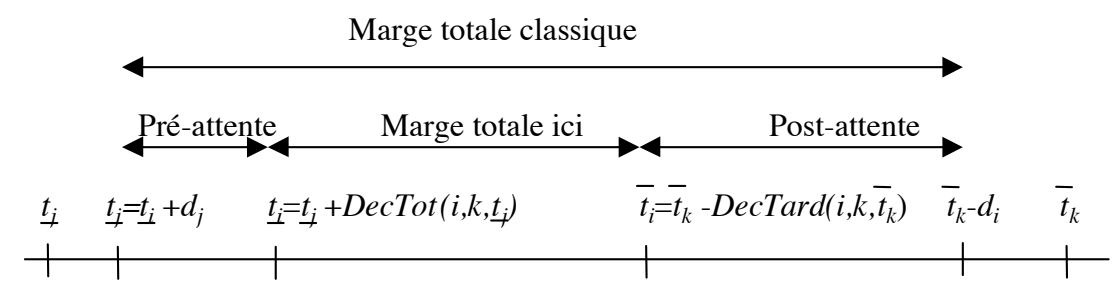

Figure 7. Expression des marges

\section{Détection et explication de l'incohérence}

A partir des ordonnancements au plus tôt et au plus tard, on peut déterminer la cohérence ou l'incohérence du problème.

Lorsque le problème est incohérent, l'objectif est de donner à l'utilisateur les causes de cet échec en vue d'une modification du problème d'ordonnancement le rendant solvable (modification du processus de production ou d'un jalon après négociation...).

\subsection{Détection de l’incohérence}

Une solution au problème de satisfaction de contraintes $P=(V, D, C)$ est une affectation de toutes les variables de $V$ dans leur domaine $D$ satisfaisant toutes les contraintes de $C$. Si une telle affectation existe (respectivement n'existe pas), on dit que le problème est cohérent (respectivement incohérent) (Verfaillie et al., 1999).

L'ordonnancement au plus tôt obtenu ne tient pas compte des contraintes de dates d'exigibilité (4). De plus, toute tâche ne peut que débuter après sa date de début au plus tôt fournie dans l'ordonnancement au plus tôt. Par suite :

- si cet ordonnancement au plus tôt respecte toutes les contraintes d'exigibilité, il est une solution du problème qui est donc cohérent ;

- s'il viole une contrainte d'exigibilité portant sur une tâche, on ne pourra pas trouver de date de début qui respecte cette contrainte. Le problème est donc incohérent.

Le problème est cohérent si et seulement si l'ordonnancement au plus tôt respecte la contrainte de dates d'exigibilité (4). De même, on montre que le problème est cohérent si et seulement si l'ordonnancement au plus tard respecte la contrainte de début du projet [1]. 


\subsection{Causes de l'incohérence}

Dans un problème de satisfaction de contraintes $\mathrm{P}=(\mathrm{V}, \mathrm{D}, \mathrm{C})$, une cause (ou explication, ou Nogood) d'incohérence ou de contradiction est un sous-problème de P noté $\mathrm{P}^{\prime}=\left(\mathrm{V}^{\prime}, \mathrm{D}, \mathrm{C}^{\prime}\right)$ lui même incohérent tel que $\mathrm{V}^{\prime} \subset \mathrm{V}$ et $\mathrm{C}^{\prime} \subset \mathrm{C}$. Une cause $\mathrm{P}^{\prime}$ sera dite minimale si tout sous-problème de $\mathrm{P}$ ' est cohérent (Jussien, 1997).

L'incohérence d'un problème peut ainsi avoir plusieurs causes distinctes (chacune étant individuellement une source de l'incohérence). Pour «réparer» un problème incohérent il faut détecter puis résoudre toutes les causes.

\subsubsection{Définition des causes de l'incohérence : chemins incohérents}

L'incohérence du problème peut être identifiée à partir des dates de début au plus tôt et des contraintes de dates d'exigibilité. Considérons un sommet (i) sur lequel porte un jalon $J_{i}-d_{i}$ et un chemin $P_{\alpha}(0, i)$ entre les sommets $(0)$ et $(i)$.

Si la longueur au plus tôt du chemin dépasse le jalon : $L t P_{\alpha}(0, i, 0)>J_{i}-d_{i}$. Alors, la date de début au plus tôt $\underline{t_{i}}$ aussi car : $\underline{t}_{\underline{i}}=\operatorname{LtP}_{P t c(0, i)}(0, i, 0) \geq L t P_{\alpha}(0, i, 0) \geq J_{i}-d_{i}$. Ainsi le chemin constitue un sous-problème incohérent. C'est donc une cause de l'incohérence. Si un des arcs appartenant à la chaîne $\alpha$ est supprimé, le chemin $P_{\alpha}(0, i)$ n'existe plus et le sous-problème résultant n'est plus incohérent. Par contre, certaines contraintes de ressources qui n'induisent aucun ou peu de décalage dans le calcul de $L t P_{\alpha}(0, i, 0)$ peuvent être supprimées de $P_{\alpha}(0, i)$ tout en conservant un sousproblème incohérent. Le chemin identifié n'est donc pas une cause minimale d'incohérence. Réciproquement, si la longueur au plus tôt du chemin $\mathrm{P}_{\alpha}(0, \mathrm{i})$ ne dépasse pas le jalon : $\operatorname{Lt}_{\alpha}(0, i, 0) \leq J_{i}-d_{i}$. Alors, le problème réduit à ce chemin est cohérent car :

$$
\underline{t_{i}}=\operatorname{Lt} P_{\alpha}(0, i, 0) \leq J_{i}-d_{i} .
$$

L'ensemble des causes de l'incohérence d'un problème de BRPSP est donc l'ensemble des chemins $P_{\alpha}(0, i)$ entre le sommet $(0)$ et un sommet $(i)$ tel qu'un jalon porte sur le sommet $(i)$ et que $L t P_{\alpha}(0, i, 0)>J_{i}-d_{i}$.

\subsubsection{Recherche des causes de l'incohérence}

Pour le calcul des dates de début au plus tôt des tâches, on ne mémorise qu'un chemin (le plus long) menant à chaque tâche. Pour exprimer l'ensemble des causes d'une incohérence, nous devons mémoriser l'ensemble des chemins et donc l'ensemble des dates de début qui mènent au dépassement d'un jalon. 
Soit $L_{j}$ la liste des dates de début calculées pour le sommet $(j)$ classée en ordre décroissant. La procédure AjouteListe $\left(L j, t, t^{\prime}, i\right)$ ajoute la date $t$ dans la liste $L j$ selon le classement voulu en mémorisant qu'elle provient de la date $t^{\prime}$ du sommet $(i)$.

0: initialisation $L_{0}=\{0\}$ and $L_{i}=\{\}$ pour $i>0$.

1: Calcul des dates de début au plus tard $\overline{t_{j}}$ en considérant la contrainte [1] relachée.

Pour tout sommet $(i)$ dans l'ordre topologique inverse

$$
\overline{t_{i}}=\operatorname{Min}_{j \in \operatorname{Succ}(i)}\left(\overline{t_{j}}-\operatorname{DecTard}\left(i, j, \overline{t_{j}}\right), J_{i}-d_{i}\right)
$$

Fin Pour

2: Si $\overline{t_{0}} \geq 0$, STOP le problème est consistent

3 : Pour tout sommet $(i)$ dans l'ordre topologique

Pour toute date $t \in L_{i}$

Pour tout sommet $(j) \in \operatorname{Succ}(i)$

$$
\mathrm{Si}\left(t+\operatorname{Dec} \operatorname{Tot}(i, j, t)>\overline{t_{j}}\right)
$$

FinPour

AjouteListe $(L j, t+\operatorname{DecTot}(i, j, t), t, i)$

FinPour

FinPour

Figure 8. Algorithme de recherche des chemins incohérents

L'algorithme proposé repose sur le principe suivant. Dans une première phase, la date de début au plus tard de chaque tâche est calculée. L'incohérence est alors déterminée par le viol de la contrainte [1]. Ensuite, un calcul similaire à celui des dates de début au plus tôt est effectué.

Toutefois, pour chaque sommet, dans l'application de la relation [12], on ne mémorise plus la valeur maximale obtenue mais toutes les valeurs calculées qui dépassent la date de début au plus tard. En effet, celles-ci et seulement celles-ci mèneront au viol d'un jalon. On mémorise ainsi par sommet, une liste de dates classées par ordre décroissant ainsi que pour chaque date la date du sommet prédécesseur l'ayant justifiée. On peut ainsi pour chaque date retrouver le chemin incohérent qui l'a justifiée.

Sur l'exemple, après la phase 1 de l'algorithme, on obtient les dates de début au plus tard comme présenté sur la figure 6. La figure 9 montre le résultat de l'algorithme. Les dates barrées sont les dates éliminées car ne dépassant pas la date de début au plus tard. 


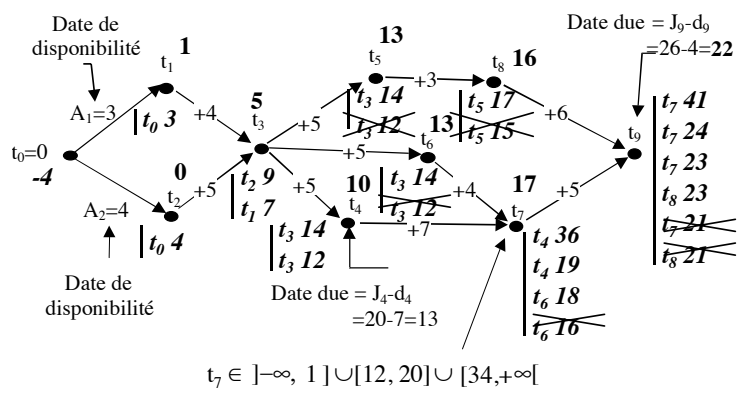

Figure 9. Exemple calcul des dates au plus tôt concernées par l'incohérence

Dans un graphe sans cycle comme supposé ici, le nombre de chemins entre le sommet source et leurs autres sommets est potentiellement en o(I!). Le temps de calcul de l'algorithme proposé est donc potentiellement en o(I !) si tous les chemins violent un jalon.

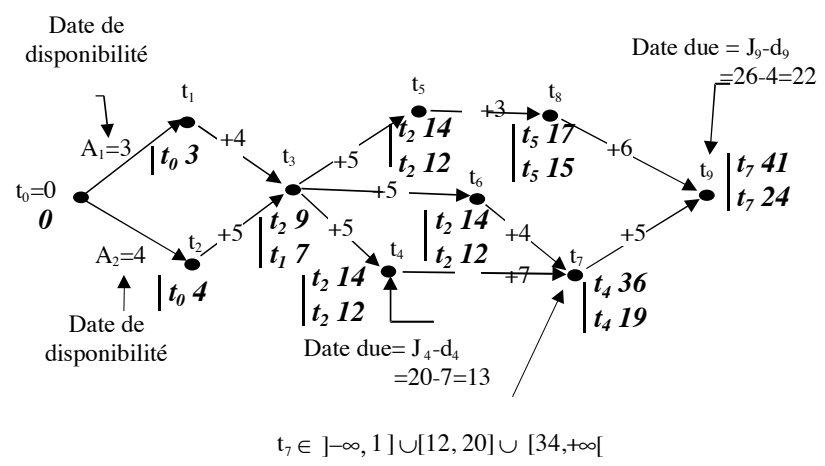

Figure 10. Exemple de limitation de la taille de la liste

On peut, par contre, limiter la taille de la liste $L_{i}$ à une valeur donnée $L$ (sur la figure $10, \mathrm{~L}=2$ ), ce qui permet de garantir que l'algorithme est en $O(I . I . L)$. Seuls les chemins les plus longs seront calculés, ce qui signifie que seules les causes majeures seront obtenues.

En utilisant ces listes, on reconstitue alors les chemins incohérents comme cela est illustré, pour le second chemin incohérent menant à t $t_{9}$ (avec une date de début au plus tôt égale à 24), sur la figure 11 . 


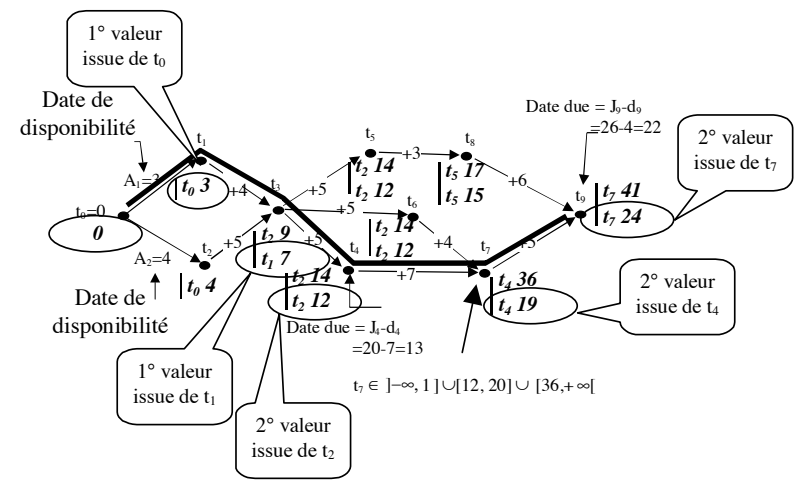

Figure 11. Exemple de chemin incohérent

On obtient ainsi 3 chemins incohérents (t4-t3-t2-t0 ; t9-t7-t4-t3-t2-t0; t9-t7-t4-t3$\mathrm{t} 1$-t 0 ) alors qu'il en existe effectivement 5 d'après les résultats de la figure $9: \mathrm{t} 4-\mathrm{t} 3$ t2-t0 ; t9-t7-t4-t3-t2-t0 ; t9-t7-t4-t3-t1-t0； t9-t7-t6-t3-t2-t0 ; t9-t8-t5-t3-t2-t0.

\subsection{Restauration de la cohérence}

La restauration de la cohérence du problème revient donc à modifier le problème tel qu'il a été exprimé en utilisant la connaissance sur les chemins incohérents et les marges. Cette restauration consiste en un ensemble de modifications qui mènent à un problème qui a une solution. Les actions suivantes sont des actions possibles pour cette restauration :

- les actions sur les paramètres internes et les contraintes internes :

- réduction de la durée des tâches (augmentation du nombre d'équipes...),

- reséquencer des tâches (modification d'un arbitrage lié à l'utilisation de la même ressource disjonctive par plusieurs tâches ; processus alternatif),

- morceler certaines tâches pour faire apparaître de nouvelles contraintes ;

- les actions sur les paramètres externes (négociation avec l'environnement)

- réduire une date d'arrivée des approvisionnements (fournisseurs),

- augmenter des dates d'exigibilité (clients),

- modifier les créneaux occupés (négociation avec les autres projets).

Le nombre de restaurations possibles est très important et elles ne peuvent donc pas être toutes listées au décideur. De plus, on ne peut pas prévoir a priori l'impact d'une modification sur les décalages liés aux ressources et donc sur la planification du réseau d'activité. Il faut simuler son impact sur le réseau. Le choix d'une procédure de restauration automatique devient ainsi difficilement envisageable. Nous 
proposons de laisser la décision au décideur en fonction des informations données par l'outil. Nous lui proposons les suivantes :

- l'intersection de tous les chemins incohérents,

- les groupes de chemins incohérents liés à la même date de disponibilité et à la même date due,

- l'ensemble des tâches appartenant à plusieurs chemins incohérents du graphe ou d'un groupe de chemins incohérents (intersections).

Sur un chemin incohérent, nous avons remarqué que toutes les tâches n'ont pas la même marge totale. Le décideur sera donc tenté de faire porter en priorité les modifications sur les tâches de marge totale les plus petites en valeur absolue. Il cherchera aussi à éliminer les pré-attentes et post-attentes qui sont des pertes de marge sans valeur ajoutée pour le projet.

\section{Application industrielle}

Cette étude a donné lieu à un prototype, qui a été développé, et testé sur des données industrielles dans le contexte d'une collaboration avec l'entreprise Astrium qui conçoit et assemble des produits complexes à la commande. Cette collaboration s'inscrit dans le cadre de l'étude du groupe DIDOM : DIstribution de la Décision dans les Organisations Matricielles (DIDOM, 2000), dont les travaux ont été soutenus par le Ministère de l'Enseignement Supérieur et de la Recherche, la Région Midi-Pyrénées, et le CNRS (programme PROSPER MPM). Ces tests ont permis de valider les algorithmes présentés ci-dessus et de montrer l'intérêt d'un tel outil dans le cadre d'une gestion distribuée.

\subsection{Contexte industriel}

Astrium conçoit et assemble des satellites. Le site étudié est la division assemblage, intégration et essais (production), dont la production est caractéristique d'une gestion multiprojet. La figure 12 présente un processus d'intégration d'un satellite (Telecom 2).

La division AIE possède une organisation matricielle basée sur trois axes de responsabilité : les projets, les ressources métiers (ressources humaines) et les ressources matérielles lourdes. Les «responsables ressources matérielles » gèrent à moyen terme ces ressources dites lourdes. Les «responsables métiers» gèrent à court terme le planning du personnel du métier en question et les « responsables projet» (ou responsables produit) gèrent leur projet. L'outil d'aide à la replanification proposé ici est donc destiné aux «responsables projet» qui sont en liaison avec les responsables des ressources matérielles. Etant donné que ce problème multiprojet est naturellement distribué, chaque centre projet est autonome et se doit d'utiliser au mieux son autonomie afin de s'adapter à son environnement. 
Pour cette raison, une approche locale du problème de planification à moyen terme en gestion multiprojet paraît particulièrement adaptée.

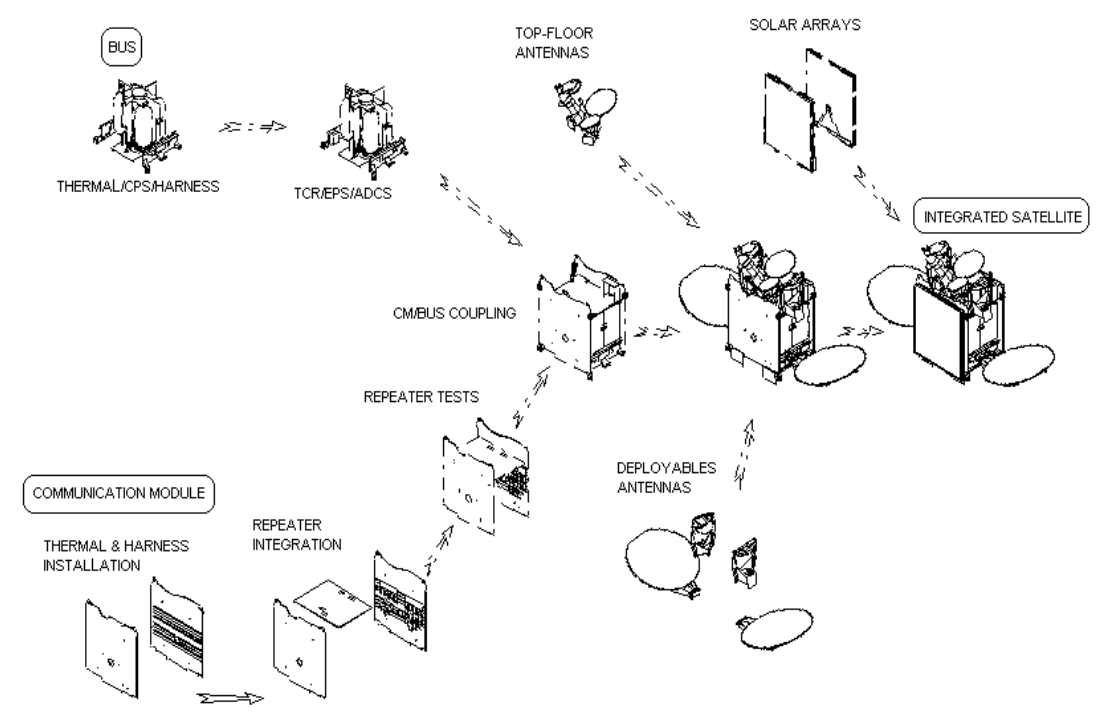

Figure 12. Processus d'intégration d'un satellite

Le responsable du projet dispose d'un problème initial (un ensemble de tâches utilisant des ressources, une séquence d'enchaînement des tâches) qui définit un problème d'ordonnancement, mais on sait que ces données pourront être modifiées lorsque des aléas entraîneront la non-existence d'une solution à ce problème initial. Dans ce contexte, le besoin d'outils d'aide pour la replanification a été identifié dans le cadre du projet DIDOM.

\subsection{Présentation des résultats du prototype}

Lorsque le problème devient incohérent, le prototype de l'outil d'aide à la replanification d'un projet dans un contexte multiprojet propose au décideur les graphes ci-après (figures 13 et 14). Le premier écran permet au décideur d'identifier tous les chemins incohérents du graphe (les groupes de chemins incohérents sont identifiés). 


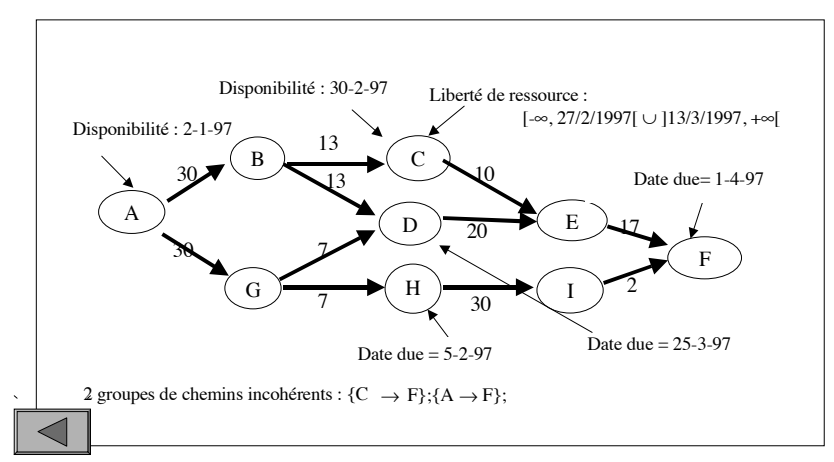

Figure 13. Interface de l'outil (chemins incohérents)

Un graphe plus précis permet pour chaque groupe de chemins incohérents de visualiser un ou plusieurs chemins incohérents et comptabilise le nombre de chemins incohérents auquel chaque arc appartient. On peut aussi sur cet écran accéder à chacun des chemins incohérents du groupe.

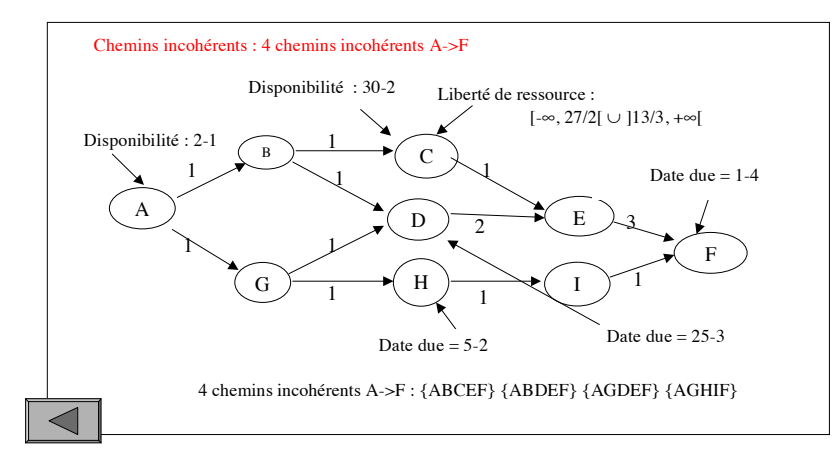

Figure 14. Groupes de chemins incohérents

Lorsque la cohérence du problème a été restaurée, des diagrammes de Gantt avec les occupations de ressources et les marges calculées sont accessibles. Ils présentent l'ordonnancement au plus tôt et le planning au plus tard.

\subsection{Tests}

L'outil d'aide à la replanification a été testé en considérant plusieurs problèmes issus d'un problème type de la division d'assemblage, intégration et essais de satellites d'Astrium. Au niveau de décision concernée, le planning d'intégration 
étudié comporte environ 60 tâches, et 100 contraintes de précédence, de dates de disponibilité et de dates d'exigibilité. Deux séquences d'intégrations possibles ont été étudiées avec des variations sur les dates d'exigibilité et les créneaux de liberté des ressources (variation du nombre de ressources): ceci définit 43 problèmes différents (Galvagnon, 2000). Deux types d'indicateurs ont été étudiés :

- le temps de calcul,

- le nombre de chemins incohérents.

\subsubsection{Temps de calcul}

Le temps de calcul, concernant la détection de l'existence ou non de l'incohérence est inférieur à 1 seconde. Le calcul des dates de début au plus tôt, dure entre 1'50 et 3'30 pour les 43 problèmes étudiés. Ces temps correspondent à une implémentation où la taille des listes n'est pas limitée : toutes les valeurs possibles des dates au plus tôt de toutes les tâches sont calculées. En limitant la taille des listes, on limiterait également le temps de calcul de ces listes.

Lorsque ces listes de dates de début au plus tôt sont construites, on reconstruit les chemins incohérents. Le temps de recherche dépend du nombre des chemins incohérents qu'il faut reconstruire. Les temps de recherche de moins d'un millier de chemins incohérents sont très raisonnables (puisque de moins de 4 minutes). Le décideur pourra donc limiter, si besoin est, le nombre de chemins reconstruits selon le temps qu'il désire accorder à l'outil.

\subsubsection{Caractéristiques des problèmes testés}

Pour mettre en exergue l'influence des paramètres caractéristiques des problèmes sur les résultats, il est nécessaire de dégager les propriétés de ces problèmes.

Les paramètres suivants, qui peuvent être calculés a priori, ont été étudiés :

- la marge du projet a priori (sans contraintes de ressources) : marge du projet au sens classique du PERT ;

- la dureté des ressources : la dureté d'une ressource est la quantité de ressource utilisée par le projet (exprimée en durée) sur le temps libre de la ressource pendant la durée du projet. Au niveau du projet, deux mesures de dureté ont été considérées : la dureté maximum et la dureté moyenne sur l'ensemble des ressources utilisées par le projet.

\subsubsection{Résultats}

Sur la figure 15, chaque point (noté *) représente un problème ou un regroupement des 43 problèmes étudiés en fonction de la marge a priori sans tenir compte des ressources, de la dureté maximale des ressources et du nombre de chemins incohérents exhibés. On retrouve des résultats quant à la sensibilité du projet au manque de marge et à la dureté des ressources : le nombre de chemins incohérents croît toujours, voire exponentiellement, lorsque la marge diminue et 
lorsque la dureté maximale des ressources croît. Il faut atteindre certains niveaux de marge pour que l'effet de la dureté des ressources soit ressenti.

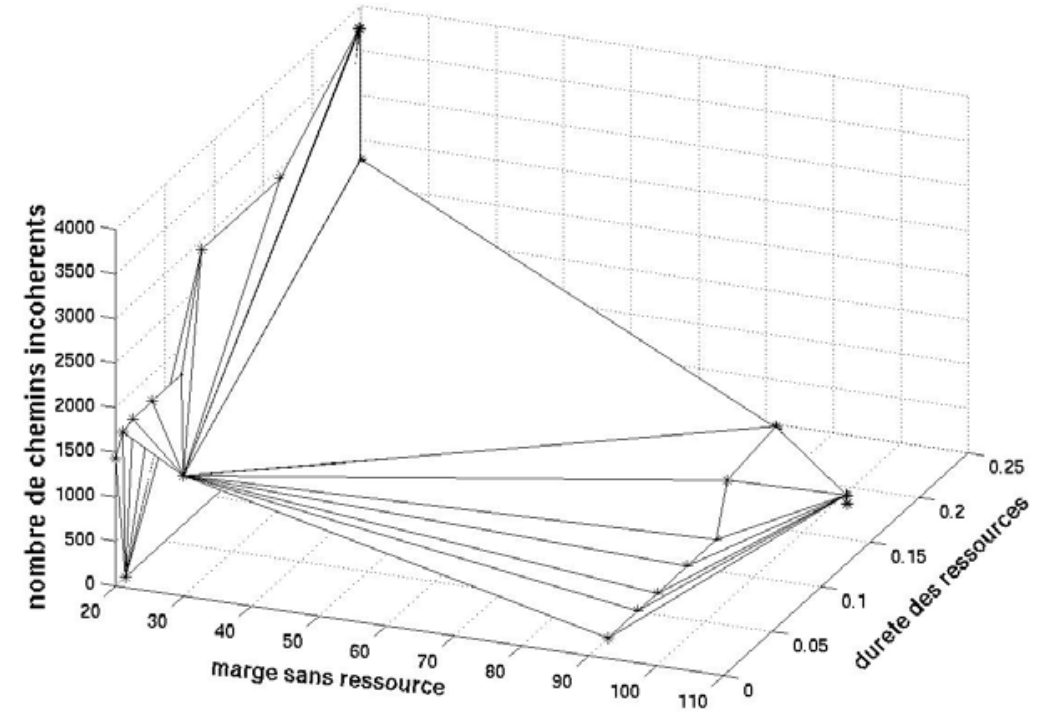

Figure 15. Influence de la marge, de la dureté des ressources sur le nombre de chemins incohérents

Un deuxième intérêt de cette courbe est de montrer que des niveaux relativement faibles d'encombrement des ressources (de 5 à $20 \%$ de leur temps disponible) suffisent à rendre fortement incohérents des projets, même pour une valeur élevée de la marge. Ceci prouve le besoin d'intégrer la considération des ressources critiques dès la planification moyen terme du projet.

Un troisième enseignement résulte de la discontinuité de la surface constatée. Lorsque pour une marge donnée la dureté maximale des ressources augmente, le nombre de chemins incohérents augmente par pallier. Ceci montre qu'il existe des effets de seuil dans la sensibilité d'un projet à la dureté des ressources. De même pour une dureté donnée, on remarque des effets de seuil lorsque la marge décroît. Une négociation de dureté des ressources n'a ainsi d'intérêt que lorsque le point est proche d'un tel seuil.

Enfin la notion de dureté maximale se révèle plus pertinente que la dureté moyenne pour l'étude de l'impact de la dureté des ressources. La surface calculée sur la dureté moyenne ne présente pas cette régularité de croissance du nombre d'incohérence et fait apparaître des trous ou des puits. On retrouve ici l'effet de la ressource «goulot» qui impose ses contraintes au projet. 


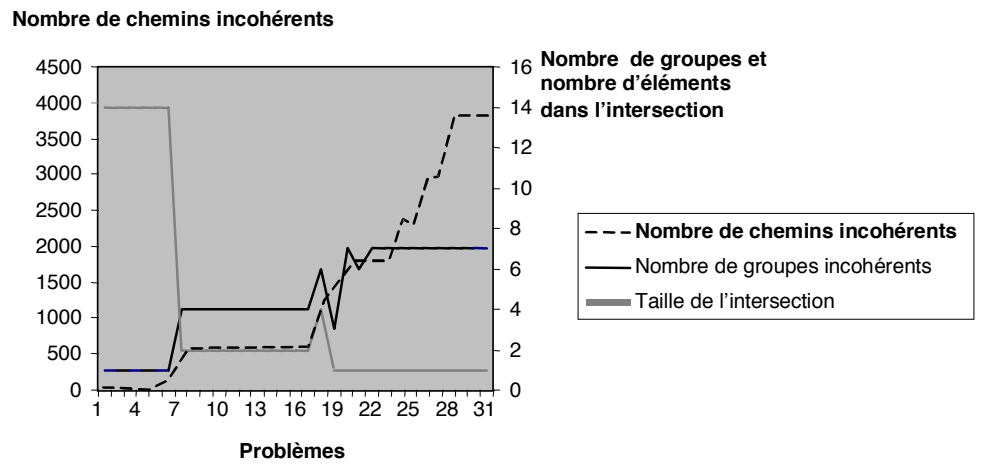

Figure 16. Comparaison entre le nombre de chemins incohérents, le nombre de groupe de chemins incohérents et la taille de l'intersection

Par ailleurs, une étude sur l'aide à l'analyse des chemins incohérents a été effectuée en considérant les paramètres suivants : le nombre de groupes de chemins incohérents constatés (définis à la section 4.3) et le nombre de tâches appartenant à l'intersection de tous les chemins incohérents. Ainsi, il apparaît (figure 16) que plus les chemins incohérents sont nombreux, plus le nombre de groupes d'incohérence est élevé, mais plus ils sont variés et plus leur intersection est petite. On note que rapidement cette intersection se résume à une tâche (la tâche fictive représentant le début du projet) ou deux. Il apparaît donc difficile de jouer sur une seule tâche pour «réparer» le problème. Par contre, le nombre de groupes incohérents reste très raisonnable (au maximum 7 dans notre cas). Ceci explique l'utilité d'aborder le problème de l'analyse et la réparation des chemins incohérents par groupe d'incohérence.

\subsubsection{Conclusion}

Les résultats de ces tests ont montré que cet outil d'aide pouvait être utilisé sur le cas industriel puisque les temps de calcul sont raisonnables ou peuvent se ramener à des temps raisonnables. En effet, lorsque ces temps de calcul deviennent prohibitifs pour une utilisation «on-line », il est possible de limiter la recherche à un certain nombre de causes de l'incohérence ce qui permet d'obtenir les causes les plus importantes en un temps limité. De plus, cette limitation du nombre de causes recherchées aura également l'intérêt de pouvoir trouver une intersection des chemins incohérents plus grande qui aidera à trouver plus facilement des actions à envisager.

\section{Conclusion et perspectives}

Nous avons présenté un outil d'aide à la replanification moyen terme d'un projet pour le responsable d'un projet en contexte multiprojet, multi-entreprise. Après avoir introduit cette approche, précisé les hypothèses du problème, nous en avons donné 
une modélisation dans laquelle les ressources partagées sont prises en compte sous la forme de créneaux de ressources réservés. Nous avons présenté une méthode dérivée de la méthode PERT, et un outil, permettant de déterminer la cohérence ou l'incohérence du problème, en se basant sur le calcul des ordonnancements au plus tôt et au plus tard que nous avons généralisés au cadre considéré. Lorsque le problème est incohérent (il est impossible de satisfaire toutes les contraintes), nous proposons alors une méthode de recherche des causes de cette incohérence sous la forme de chemins incohérents dans le graphe représentant le projet avec réservation de créneaux temporels de ressources. De plus, l'utilisateur pourra bénéficier d'une analyse du graphe basée sur les deux ordonnancements précités: en termes de marges, tâches critiques, chemins critiques mais aussi de groupes d'incohérence, d'intersections de chemins incohérents, d'édition des chemins incohérents.

Le problème industriel dont s'inspirent ces travaux a ensuite été présenté : il s'agit du problème de gestion de l'intégration d'un satellite dans la division Assemblage, Intégration et Essais de la société Astrium. Les composants du problème type ont été exhibés et ont servi à définir un ensemble de problèmes sur lesquels le prototype de l'outil d'aide a été testé.

A partir de cette étude nous avons pu mettre en avant l'intérêt :

- de développer l'aspect analyse du graphe après l'ordonnancement afin de fournir une interface utilisateur correspondant aux attentes des responsables projets,

- d'améliorer la partie concernant l'explication de l'incohérence notamment pour fournir des informations plus précises à l'utilisateur.

Enfin, une de nos priorités est l'aide au responsable de projet dans l'exploitation des causes de l'incohérence détectées pour restaurer la cohérence du problème et pouvoir trouver une solution. La longueur d'un chemin dépendant de la date à laquelle il est considéré, les marges étant variables le long d'un chemin, les méthodes d'aide à la réparation par optimisation nous semblent difficilement exploitables. Nous pourrons par contre nous appuyer sur des travaux du groupe DIDOM (DIDOM, 2000), notamment sur une analyse sociologique du processus de planification dans l'entreprise.

Dans la continuité de ces travaux d'autres études sont actuellement menées. Le modèle de planification a été étendu pour intégrer des durées de production incertaines et donc de créneaux de disponibilité également (Fargier et al., 2002). Par ailleurs, en présence de données incertaines, une autre approche (la plus répandue) consiste à utiliser des procédures à horizon glissant qui permettent de mettre à jour ces données au cours du temps. Une extension de la méthode de recherche des causes de l'incohérence ainsi que des actions possibles pour restaurer la cohérence est en cours d'étude pour exploiter le processus dynamique de planification à horizon glissant (Thierry et al., 2001). 


\section{Bibliographie}

Boctor F.F., «A new and efficient heuristic for scheduling projects with resource restrictions and multiple execution modes », European Journal of Operational Research, vol. 90, $\mathrm{n}^{\circ} 2$, p. 349-361, 1996.

Brucker P., Drexl, A., Möhring R., Neumann K., E. Pesch, «Resource-constrained Project scheduling: Notation, Classification, Models, and Methods », European Journal of Operational Research, vol. 112, n 1, p. 3-41, 1999.

Chen Y-L, Rinks D., Tang K., "Critical path in an activity network with time constraints », European Journal of Operational Research, vol. 100, p. 122-133, 1997.

Crowston W., «Decision CPM : network reduction and solution», Operations Research Quaterly, vol. 21, n 4, p. 435-445, 1971.

Dauzère-Péres S., Lasserre J.B., «An Integrated Approach in Production Planning and Scheduling », Lectures Notes in Economics and Mathematical Systems, Springer-Verlag, Berlin, 1994.

DIDOM group, «Planning by repairing and co-operation: a more realistic approach to multiproject management », MCPL 2000, Grenoble, France, 2000.

Dorndorf U., Huy T.P, Pesch E., « A survey of interval capacity tests for time -and resourceconstrained scheduling », J. Weglarz (ed), Project scheduling - Recent Models, Algorithms and Applications, Kluwer Academic Publishers, Boston, p. 213-238, 1999.

Drexl A., Kimms A., « Lot sizing and scheduling-survey and extensions », European Journal of Operational Research, 99, p. 221-235, 1997.

Elmaghraby S.E., Activity network: project planning and control by network models, Wiley, New York, 1977.

Fargier H. et Thierry C., «Evaluation du risque dans la gestion d'un projet sous contraintes de ressources externes : l'approche probabiliste », LFA, Rencontres francophones sur la logique floue et ses applications, Montpellier, France, 2002.

Galvagnon V, Thierry C., «Scheduling and restoring the consistency of a project in multiproject context », MCPL 2000, Grenoble, France.

Galvagnon V., Aide à la décision en gestion multiprojet distribuée: approche locale pour la planification à moyen terme, Thèse de doctorat, ENSAE, France, 2000.

Giard V., Gestion de projet, Edition Economica, collection Gestion, 1991.

Giard V., Midler C., Management et gestion de projet : bilan et perspectives, encyclopédie de gestion, Economica, 1996

Giard V., Gestion de la production, Edition Economica, collection Gestion, 1988.

Golenko-Ginsburg D., Blokh D., «A generalized Activity Network», Journal of the Operational Research Society, 48, p. 399-400, 1997.

Hartmann S., Kolisch R., «Experimental Evaluation of State-of-the-Art Heuristics for the Resource-Constrained Project Scheduling Problem », Manuskripte aus den Instituten für Betriebswirtschaftslehre, $\mathrm{n}^{\circ} 476,1998$. 
Herroelen W.E., Demeulemeester E.L., De Reyck B., «A classification scheme for project scheduling problems J. Weglarz (ed.), Project scheduling - Recent Models, Algorithms and Applications, Kluwer Academic Publishers, Boston, Chapitre 1, 1999.

Jussien N., «Relaxation de Contraintes pour les problèmes dynamiques », Thèse de doctorat, Université de Rennes I, France, 1997.

Kelley J.E., «The critical path planning and scheduling: Mathematical basis », Operations Research, 9, p. 296-320, 1961.

Kolisch R., Make-to-order Assembly Management, Springer 2001.

Kolisch R, Padman R., «An Integrated Survey of deterministic project scheduling. OMEGA », International Journal of Management Science, vol. 29, n 3, p. 249-272, 2001.

Kolisch R., Hartmann S., «Heuristic algorithms for the resource-constrained project scheduling problem : classification and computational analysis », J. Weglarz (ed.), Project scheduling - Recent Models, Algorithms and Applications, Kluwer Academic Publishers, Boston, p. 147-178, 1999.

Kurtulus I., Narula S., «Multi-project scheduling, Analysis of Project Performance », IIE Transactions, vol. 17, $\mathrm{n}^{\circ} 1$, p. 58-66, 1985.

Maes J., McClain J., Van Wassenhove L., «Multilevel capacitated lotsizing complexity and LP-based heuristics », European Journal of Operational Research, vol. 53, 1991.

Nuijten W.P., Time resource Constrained Scheduling: A constraint Satisfaction Approach, PhD Thesis, Eindhoven University of technology, 1994.

Project Management Institute, A guide to the project management Body of Knowledge (PMBOK), PMI, 1994

Salewskia F., Schirmera A., Drexl A., «Project scheduling under resource and mode identity constraints: Model, complexity, methods, and application », European Journal of Operational Research, vol. 102, n 1, p. 88-110, 1997.

Speranza M. G., Vercellis C., «Hierarchical models for multi-project planning and scheduling », European Journal of Operational Research, vol. 64, p. 312-325, 1993.

Thierry C., Lamothe J., Galvagnon V., « Outil d'aide à la décision pour la planification d'un projet en environnement multiprojet », $4^{e}$ Congrès international de Génie Industriel, Aix, France, 2001.

Verfaillie G., Lobjois L., "Problèmes incohérents: expliquer l'incohérence, restaurer la cohérence », $5^{e}$ Journées Nationales sur la Résolution Pratique de Problèmes NP. Complets (JNPC-99), Lyon, France, 1999.

Yang K., Sum C., «An evaluation of due date, resource allocation, project release, and activity scheduling rules in a multiproject environment», European Journal of Operational Research, vol. 103, p. 139-154, 1997.

Weglarz J. (ed.)., Project scheduling - recent models, algorithms and applications, Kluwer Academic Publishers, Boston 1999. 